\title{
Light scalar production from Higgs bosons and FASER 2
}

\author{
Iryna Boiarska, ${ }^{a}$ Kyrylo Bondarenko, ${ }^{b}$ Alexey Boyarsky, ${ }^{b}$ Maksym Ovchynnikov, ${ }^{b}$ \\ Oleg Ruchayskiy ${ }^{a}$ and Anastasia Sokolenko ${ }^{c}$ \\ ${ }^{a}$ Discovery Center, Niels Bohr Institute, Copenhagen University, \\ Blegdamsvej 17, DK-2100 Copenhagen, Denmark \\ ${ }^{b}$ Intituut-Lorentz, Leiden University, \\ Niels Bohrweg 2, 2333 CA Leiden, The Netherlands \\ ${ }^{c}$ Department of Physics, University of Oslo, \\ Box 1048, NO-0371, Oslo, Norway \\ E-mail: boiarska@nbi.ku.dk, bondarenko@lorentz.leidenuniv.nl, \\ boyarsky@lorentz.leidenuniv.nl, ovchynnikov@lorentz.leidenuniv.nl, \\ oleg.ruchayskiy@nbi.ku.dk, anastasia.sokolenko@fys.uio.no
}

ABSTRACT: The most general renormalizable interaction between the Higgs sector and a new gauge-singlet scalar $S$ is governed by two interaction terms: cubic and quartic. The quartic term is only loosely constrained by invisible Higgs decays and given current experimental limits about $10 \%$ of all Higgs bosons at the LHC can be converted to new scalars with masses up to $m_{\text {Higgs }} / 2$. By including this production channel, one significantly extends the reach of the LHC-based Intensity Frontier experiments. We analyze the sensitivity of the FASER experiment to this model and discuss modest changes in the FASER 2 design that would allow exploring an order-of-magnitude wider part of the Higgs portal's parameter space.

Keywords: Beyond Standard Model, Higgs Physics

ArXiv EPrint: 1908.04635 


\section{Contents}

1 Introduction: scalar portal and FASER experiment 1

1.1 Existing bounds 3

1.2 The FASER experiment 3

2 Scalars from Higgs bosons $\quad \mathbf{5}$

2.1 Naive estimate: what can be expected? 5

2.2 Geometrical acceptance 6

2.3 Decay of scalars 8

$\begin{array}{llr}3 & \text { Results } & 9\end{array}$

4 Conclusion $\quad 11$

$\begin{array}{ll}\text { A Higgs boson distribution } & 13\end{array}$

$\begin{array}{ll}\text { B Distributions } & \mathbf{1 4}\end{array}$

$\begin{array}{lll}\text { B.1 Kinematics in laboratory frame } & 14\end{array}$

$\begin{array}{lll}\text { B.2 Distribution of scalars over energies and polar angles } & 15\end{array}$

\section{Introduction: scalar portal and FASER experiment}

The Standard Model of particle physics (SM) is extremely successful in explaining accelerator data. Yet it fails to explain several observed phenomena: neutrino masses, dark matter and baryon asymmetry of the Universe. To explain these phenomena, we need to postulate new particles that should not nevertheless spoil extremely successful Standard Model predictions. These new hypothetical particles can be heavy, thus evading detection at $\sqrt{s}=13 \mathrm{TeV}$ collision energy of the LHC. Such particles would induce higher-dimensional (non-renormalizable) interactions with SM fields, the signatures of such operators are being searched at the LHC (see e.g. [1] for a review).

Alternatively, new particles can be light yet have very weak couplings to the Standard Model - feebly interacting particles, or FIPs. In this case, their interaction with the SM can be governed even by relevant (dimensions 3 and 4) operators with small couplings. Such models are generically called portals because trough such operators FIPs can mediate interactions with some "dark sectors" - other new particles that otherwise are inaccessible.

In this paper, we consider the most general form of the scalar (or Higgs) portal [2-5] that has been the subject of active analysis in the recent years, see e.g. [6-9] and refs. 
therein. Namely, we introduce a scalar particle $S$ that carries no Standard Model charges and interacts with the Higgs doublet $H$ via

$$
\mathcal{L}=\mathcal{L}_{S M}+\frac{1}{2}\left(\partial_{\mu} S\right)^{2}+\left(\alpha_{1} S+\alpha_{2} S^{2}\right)\left(H^{\dagger} H-\frac{v^{2}}{2}\right)-\frac{m_{S}^{2}}{2} S^{2}
$$

where $v$ is the Higgs VEV and the model is parametrized by three new constants: $\alpha_{1}, \alpha_{2}$ and the scalar mass $m_{S}$. After electroweak symmetry breaking, the $S H H$ interaction (1.1) leads to a quadratic mixing between $S$ and the Higgs boson $h$. Transforming the Higgs field into the mass basis, $h \rightarrow h+\theta S(\theta \ll 1)$, one arrives at the following Lagrangian, describing interactions of the new boson $S$ with the SM fermions, intermediate vector bosons and the Higgs boson:

$$
\mathcal{L}_{S M}^{S}=-\theta \frac{m_{f}}{v} S \bar{f} f+2 \theta \frac{m_{W}^{2}}{v} S W^{+} W^{-}+\theta \frac{m_{Z}^{2}}{v} S Z^{2}+\frac{\alpha}{2} S^{2} h+\ldots
$$

where ... denote quartic and higher terms. The interactions (1.2) also mediate effective couplings of the scalar to photons, gluons, and flavor changing quark operators [10], opening many production channels at both LHC and Intensity Frontier experiments. The phenomenology of light GeV-like scalars has been worked out in [11-21] as well as in [22-31] in the context of the light Higgs boson. Most of these works concentrated on the Lagrangian with $\alpha_{1}=0$ in which case the couplings $\theta$ and $\alpha$ in (1.2) become related. ${ }^{1}$ In this work we consider $\alpha_{1} \neq 0$. Phenomenologically, this allows to decouple decay channels (controlled by $\theta$ ) and production channels (controlled by $\alpha$ ), cf. [33] where phenomenology of such a model is also discussed. As we will see below, the parameter $\alpha$ is only weakly constrained by the invisible Higgs decays $[34,35]$ and can be quite sizeable (if unrelated to $\theta)$. As a result, the production via $h \rightarrow S S$ process becomes possible and is operational for scalar masses up to $m_{h} / 2$ which allows to significantly extend the sensitivity reach of the LHC-based experiments.

We note that the production channel via the off-shell Higgs bosons (e.g. coming from neutral meson decays, such as $B_{s} \rightarrow S S$ for $2 m_{S}<m_{B}$ ) starts to dominate over production via flavour changing mixing for $\theta^{2}<10^{-9} \div 10^{-10}$, see [10]. We will not consider this effect in the current work, mostly concentrating on $m_{S} \gtrsim 5 \mathrm{GeV}$.

Searches for light scalars have been previously performed by CHARM [36], KTeV [37], E949 [38, 39], Belle [40, 41], BaBar [42], LHCb [43, 44], CMS [34, 45, 46] and ATLAS [35, 47-49] experiments. Significant progress in searching for light scalars can be achieved by the proposed and planned intensity-frontier experiments such as SHiP [8, 50, 51], CODEXb [52], MATHUSLA [16, 51, 53, 54], FASER [55, 56], SeaQuest [57], NA62 [58-60] and a number of other experiments (see [61] for an overview). The summary of the current experimental status of the light scalar searches is provided in the Physics Beyond Collider report [61].

\footnotetext{
${ }^{1}$ Alternative class of models has super-renormalizable interaction only between the Higgs boson and the scalar $\left(\alpha_{2}=0\right)$, see [32] and refs. therein. In this case, of course, there is no $S^{2} h$ term in the Lagrangian (1.2).
} 

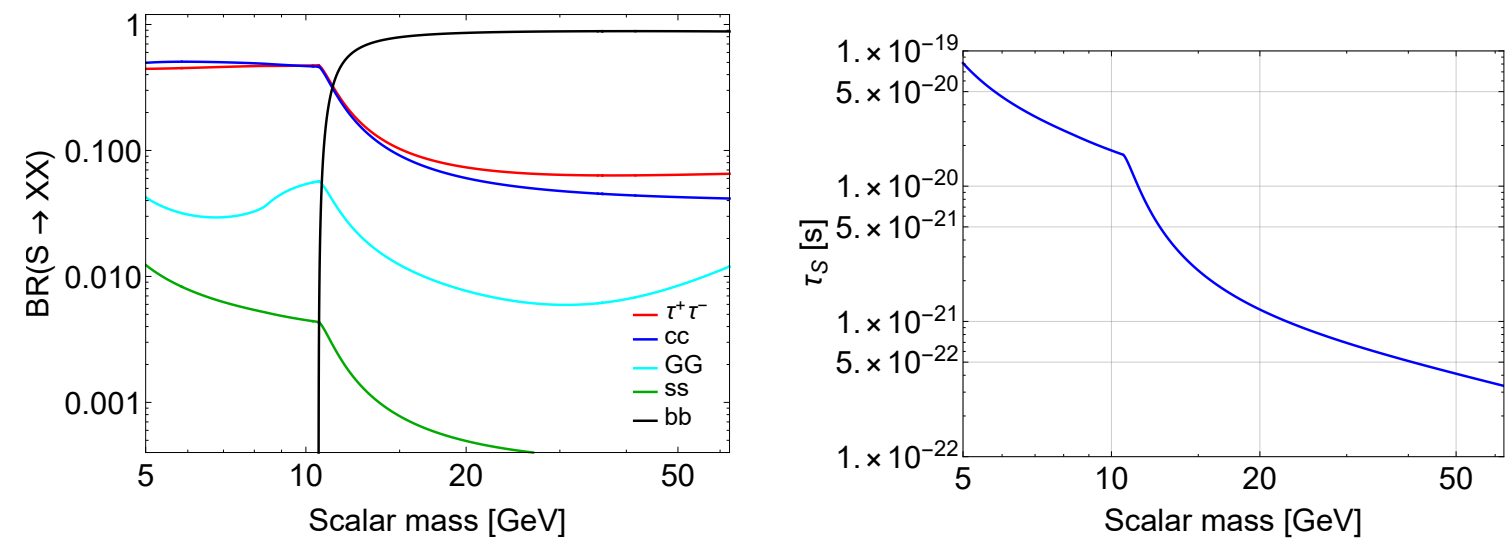

Figure 1. Left panel: branching ratios of the decays of a scalar $S$ as a function of its mass. We use perturbative decays into quarks and gluons (see [10] for details). Right panel: the lifetime of a scalar $S$ as a function of its mass for the mixing angle $\theta^{2}=1$. The lifetime is obtained using decays into quarks and gluons (and $\tau$ 's) within the framework of perturbative QCD.

\subsection{Existing bounds}

The up to date experimental constraints in the $m_{S^{-}} \theta$ plane can be found in the scalar portal section of [61]. The strongest experimental constraints on the parameter $\alpha$ come from the invisible Higgs decay. In the Standard Model the decay $h \rightarrow Z Z \rightarrow 4 \nu$ has the branching ratio $\mathcal{O}\left(10^{-3}\right)$. Current limits on the Higgs to invisible are $\mathrm{BR}_{\text {inv }}<0.19$ at $95 \%$ CL [34]. Future searches at LHC Run 3 and at the High-Luminosity (HL) LHC (HL-LHC, Run 4) are projected to have sensitivity at the level $\mathrm{BR}_{\text {inv }} \sim 0.05-0.15$ at $95 \%$ CL [62] maybe going all the way to a few percents [63]. In what follows we will assume that the branching ratio $\mathrm{BR}_{\text {inv }}$ is saturated by the $h \rightarrow S S$ decay. Using

$$
\Gamma_{h \rightarrow S S}=\frac{\alpha^{2}}{32 \pi m_{h}} \sqrt{1-\frac{4 m_{S}^{2}}{m_{h}^{2}}}
$$

we obtain the corresponding value of $\alpha^{2} \sim 5 \mathrm{GeV}^{2}$ for $m_{S} \ll m_{h}$.

Apart from the invisible Higgs decays, the ATLAS and CMS collaborations have previously performed studies of the $h \rightarrow S S \rightarrow 4 b, h \rightarrow S S \rightarrow 2 b 2 \mu, h \rightarrow S S \rightarrow 2 \tau 2 \mu$, $h \rightarrow S S \rightarrow 2 \tau 2 b$, etc. [45, 46, 48, 49,64,65] for the light (pseudo)scalar in the mass ranging between $\mathcal{O}(10) \mathrm{GeV}$ and $m_{h} / 2$. The obtained constraints, however, do not restrict the parameters relevant for the FASER 2 experiment as they search for prompt decays of the scalars, while in our model the $c \tau_{S} \sim \mathcal{O}(100)$ meters.

\subsection{The FASER experiment}

FASER (ForwArd Search ExpeRiment, figure 2), is an Intensity Frontier experiment dedicated to searching for light, extremely weakly-interacting particles that may be produced in the LHC's high-energy collisions in the far-forward region and then travel long distances without interacting [55, 66-69]. FASER is approved to collect data in 2021-2023 during the LHC Run 3. If FASER is successful, FASER 2, a much larger successor, could be 


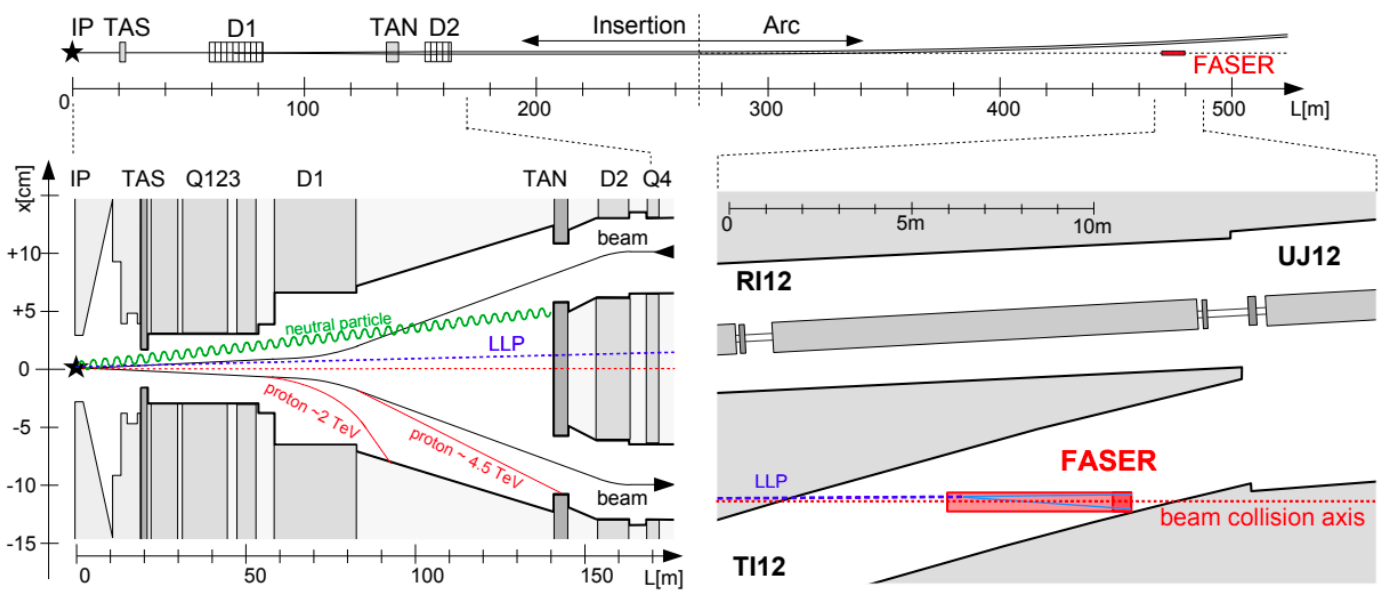

Figure 2. The scheme of the FASER experiment. The figure from [66].

\begin{tabular}{|l|c|c|c|c|c|}
\hline Phase & $\mathcal{L}, \mathrm{fb}^{-1}$ & $L[\mathrm{~m}]$ & $R[\mathrm{~m}]$ & $l_{\text {det }}[\mathrm{m}]$ & $\theta_{\text {FASER }}[\mathrm{rad}]$ \\
\hline FASER & 150 & 480 & 0.1 & 1.5 & $2.1 \cdot 10^{-4}$ \\
\hline FASER 2 & 3000 & 480 & 1 & 5 & $2.1 \cdot 10^{-3}$ \\
\hline $\begin{array}{l}\text { FASER 2 } \\
\text { (alternative configuration) }\end{array}$ & 3000 & 480 & 1.5 & 5 & $3.1 \cdot 10^{-3}$ \\
\hline
\end{tabular}

Table 1. Parameters of the FASER experiment. Prototype detector (FASER) is approved to collect data during the LHC Run 3. FASER 2 is planned for HL-LHC phase, but its configuration is not finalized yet. In the third line, we propose an alternative configuration of FASER 2 that would allow drastically increasing its reach towards the scalar portal. $\mathcal{L}$ is the integrated luminosity of the corresponding LHC run. $L$ is the distance between the ATLAS interaction point and the entrance of the FASER decay vessel. $R$ is the radius of the decay vessel. $l_{\text {det }}$ is the length of the detector and $\theta_{\mathrm{FASER}}=R / L$ is the angle, so that the solid angle subtended by the detector is given by $\Omega_{\mathrm{FASER}}=\pi \theta_{\mathrm{FASER}}^{2}$. For our investigation, we assume that the decay vessel is a cylinder, centered around the beam axis.

constructed in Long Shutdown 3 and collect data during the High-Luminosity Run 4 in 2026-2035. The relevant parameters of FASER and FASER 2 are shown in table 1. We also list the alternative configuration of FASER 2 which we will use for comparison in this work.

While the design of the first phase is fixed, the FASER 2 is not finalized yet. We demonstrate therefore how the parameters of the future FASER 2 experiment will affect its sensitivity.

The paper is organized as follows:

- In section 2 we estimate the number of decay events in the FASER detectors. This section allows for easy cross-check of our main results and gives the feeling of the main factors that affect the sensitivity.

- In section 3 we outline our estimates based on which the conclusion is drawn. We 
also demonstrate that an increase of the geometric acceptance by the factor $\sim 2$ (e.g. via increase of the radius of the decay vessel of FASER 2 from $1 \mathrm{~m}$ to $1.5 \mathrm{~m}$ ) would allow a wide region of the parameter space to be probed.

- Appendices provide some details of our computations that would permit the interested reader to reproduce them.

\section{Scalars from Higgs bosons}

\subsection{Naive estimate: what can be expected?}

Before running MC simulations (and to have a way to verify the simulation results) we start with analytic estimates of the sensitivity of FASER 2. The number of detected events is given by the following formula [51]:

$$
N_{\text {det }}=N_{S} \times \epsilon_{\text {geom }} \times P_{\text {decay }} \times \epsilon_{\text {det }} .
$$

Here, $N_{S}$ is the number of scalars produced at the LHC experiment; in our case $N_{S}=$ $2 N_{h} \operatorname{BR}(h \rightarrow S S), N_{h}$ - the number of produced Higgs bosons, $\epsilon_{\text {geom }}$ is the geometric acceptance - the fraction of scalars whose trajectories intersect the decay volume, so that they could decay inside it. The decay probability is given by the well-known formula

$$
P_{\text {decay }}\left(l_{\text {decay }}\right)=e^{-L / l_{\text {decay }}}-e^{-\left(L+l_{\text {det }}\right) / l_{\text {decay }}},
$$

where $L$ is the distance from the interaction point to the entrance of the fiducial volume, $l_{\text {det }}$ is the detector length, and $l_{\text {decay }}=c \tau_{S} \beta_{S} \gamma_{S}$ is the decay length. Finally, $\epsilon_{\text {det }} \leq 1$ is the detection efficiency - a fraction of all decays inside the decay volume for which the decay products could be detected. In the absence of detector simulations, we optimistically assume detector efficiency of FASER to be $\epsilon_{\text {det }}=1$.

The high luminosity LHC phase is expected to deliver $1.7 \cdot 10^{8}$ Higgs bosons (the Higgs boson production cross-section at $\sqrt{s}=13 \mathrm{TeV}$ is $\sigma_{h} \approx 55 \mathrm{pb}$ [70], going to $60 \mathrm{pb}$ at $14 \mathrm{TeV})$. Further, we assume the fiducial Higgs decay to scalars equal to the lower bound of HL-LHC reach [62]:

$$
\mathrm{BR}_{\mathrm{fid}}(h \rightarrow S S)=0.05
$$

For the initial estimate of the number of produced scalars, we consider these Higgs bosons decaying at rest. In this case, we estimate the number of scalars flying into the solid angle of FASER 2 as

$$
\epsilon_{\mathrm{geom}}^{\text {naive }}=\frac{\Omega_{\mathrm{FASER}}}{4 \pi} \approx 1.1 \times 10^{-6}
$$

where $\Omega_{\text {FASER }}=\pi \theta_{\text {FASER }}^{2}$, see table 1 . Plugging in the numbers we get $N_{S}^{\text {naive }}=2 N_{h} \times$ $\epsilon_{\text {geom }}^{\text {naive }} \times \mathrm{BR}_{\text {inv }} \approx 33$ scalars. As most of the Higgs bosons fly along the beam axis, eq. (2.4) is a strong underestimate and we should expect a lot of scalars flying through the FASER fiducial volume. 

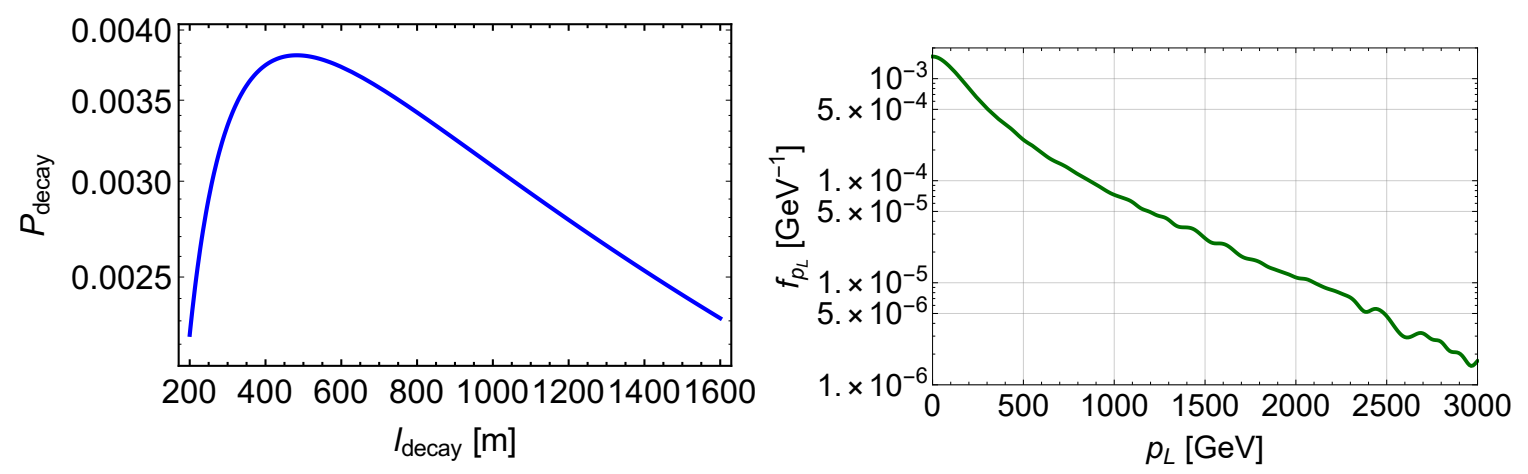

Figure 3. Left panel: A probability of the scalar decay for FASER 2 as a function of a scalar's decay length $l_{\text {decay }}$. Right panel: the distribution function $f_{p_{L}}=\frac{1}{N_{h}} \frac{d N_{h}}{d p_{L}}$ of Higgs bosons by longitudinal momentum $p_{L}$. The simulations are based on MadGraph5_aMCNLO [71] and following [72]. See appendix A for details.

For $l_{\text {det }} \ll L$ (as it is the case for FASER/FASER 2) the probability of decay (2.2) reaches its maximum for $l_{\text {decay }} \approx L$. The maximum is purely geometric, not related to the parameters of the scalar $S$ and numerically it is equal to

$$
P_{\text {decay }}^{(\max } \simeq \frac{l_{\text {det }}}{L} e^{-1} \approx 3.8 \cdot 10^{-3},
$$

see also figure $3 .^{2}$ Multiplying eqs. (2.4) and (2.5) we find $\mathcal{O}(0.1)$ detectable events. Given that this was a (strong) underestimate - we see that more careful analysis is needed. It will proceed as follows:

1. We start by assuming that all Higgs bosons travel along the beam axis, which allows for a much simplified analytic treatment. Then we comment on the effect of $p_{T}$ distribution of the Higgs bosons.

2. We determine the realistic geometrical acceptance $\epsilon_{\text {geom }} \gg \epsilon_{\text {geom }}^{\text {naive }}$, since the actual angular distribution of scalars is peaked in the direction of the FASER detector.

3. Finally, as scalars have non-trivial distribution in energy, for most of the scalars the decay probability is not equal to the maximal value, thus determining the width of the sensitivity area in the $\theta$ direction for a given mass.

\subsection{Geometrical acceptance}

Most Higgs bosons are traveling along the beam axis and therefore have $p_{T} \ll p_{L}$ (see appendix A). Therefore, we perform the analytic estimates based on the purely longitudinal distribution of the Higgs bosons $f_{p_{L}} \equiv \frac{1}{N_{h}} \frac{d N_{h}}{d p_{L}}$ shown in figure 3 .

\footnotetext{
${ }^{2}$ The independence of the value (2.5) of the mass $m_{S}$ can be understood in the following way. Since the production of the scalar is independent on the coupling $\theta^{2}$ while the decay length depends on $\theta^{2}$, we can always adjust it for a fixed mass $m_{S}$ in a way such that $l_{\text {decay }}\left(m_{S}, \theta^{2}\right)=L$. As we demonstrate below the values of $\theta^{2}$ for masses of interest (from few $\mathrm{GeV}$ to $m_{h} / 2$ ) correspond to the region of the scalar parameter space that is currently unprobed by existing experiments.
} 
The angle $\theta_{S}$ between the scalar and Higgs boson directions in the laboratory frame is related to the scalar direction in the Higgs rest frame via

$$
\tan \theta_{S}=\frac{1}{\gamma_{h}} \frac{\beta_{S}^{\prime} \sin \theta_{S}^{\prime}}{\beta_{S}^{\prime} \cos \theta_{S}^{\prime}+\beta_{h}}
$$

where

$$
\beta_{S}^{\prime}=\sqrt{1-\frac{4 m_{S}^{2}}{m_{h}^{2}}}
$$

is the velocity of a scalar in the rest frame of the Higgs boson, $\gamma_{h}$ and $\beta_{h}$ are Higgs boson's gamma factor and the velocity in the laboratory frame. ${ }^{3}$

Based on these considerations, we can calculate the geometric acceptance (once again assuming that all Higgs bosons fly in the direction of the beam):

$$
\epsilon_{\mathrm{geom}} \approx \int f_{p_{L}} \kappa\left(m_{S}, p_{L}\right) \frac{\Omega\left(p_{L}\right)}{4 \pi} d p_{L}
$$

Here, $\Omega$ is the solid angle of FASER 2 available for scalars:

$$
\Omega=\left\{\begin{array}{l}
\Omega_{\mathrm{FASER}}, \quad \theta_{\mathrm{FASER}}<\theta_{\mathrm{max}} \\
\pi \theta_{\max }^{2}\left(p_{L}\right), \quad \theta_{\mathrm{FASER}}>\theta_{\max }
\end{array}\right.
$$

with $\theta_{\max }=\arctan \left[\frac{\beta_{S}^{\prime}}{\gamma_{h} \sqrt{\beta_{h}^{2}-\beta_{S}^{\prime 2}}}\right]$ if $\beta_{h}>\beta_{S}$ and $\theta_{\max }=\pi$ otherwise. Finally, the function $\kappa=\left|d \Omega^{\prime} / d \Omega\right|$, where $\Omega$ is the solid angle in the lab frame corresponding to the solid angle $\Omega^{\prime}$ in the Higgs rest frame. It defines how collimated is the beam of scalars as compared to an isotropic distribution. For the details of the derivation of the explicit expression of $\kappa$ see appendix 2.2. In the case $\theta=0$ it becomes

$$
\kappa\left(m_{S}, p_{L}\right) \approx \begin{cases}\frac{2 \gamma_{h}^{2}\left(\beta_{S}^{\prime 2}+\beta_{h}^{2}\right)}{\beta_{S}^{\prime 2}}, & \beta_{h}>\beta_{S}^{\prime}, \\ \frac{\gamma_{h}^{2}\left(\beta_{S}^{\prime}+\beta_{h}\right)^{2}}{\beta_{S}^{\prime 2}}, & \beta_{h}<\beta_{S}^{\prime}\end{cases}
$$

The resulting acceptance (see figure 5 , left panel) grows with the mass since the maximal angle $\theta_{S}$ decreases; when the mass of the scalar is very close to $m_{h} / 2$, the acceptance reaches its maximum equal to the fraction of Higgs bosons flying into the direction of the FASER 2 decay volume, $f_{h \rightarrow \text { FASER }}$. Even for the light scalars the acceptance $\epsilon_{\text {geom }} \approx 4 \cdot 10^{-5}$ is an order of magnitude larger than the naive estimate (2.4). The reason for this is that most of the Higgs bosons have large energies, so the resulting angular distribution of scalars is peaked in the direction of small angles, see figure 4 .

With $p_{L}$ distribution only, obviously, $f_{h \rightarrow \text { FASER }}=1$. To make realistic estimates, we need to take into account the $p_{T}$ distribution of the Higgs bosons. The fraction $f_{h \rightarrow \text { FASER }}$

\footnotetext{
${ }^{3}$ Although two scalars originate from each Higgs decay, the angle between the scalars in the laboratory frame is larger than $\theta_{\text {FASER }}$ unless $m_{S}$ is very close to $\frac{m_{h}}{2}$. In appendix B.2 we provide detailed estimates.
} 


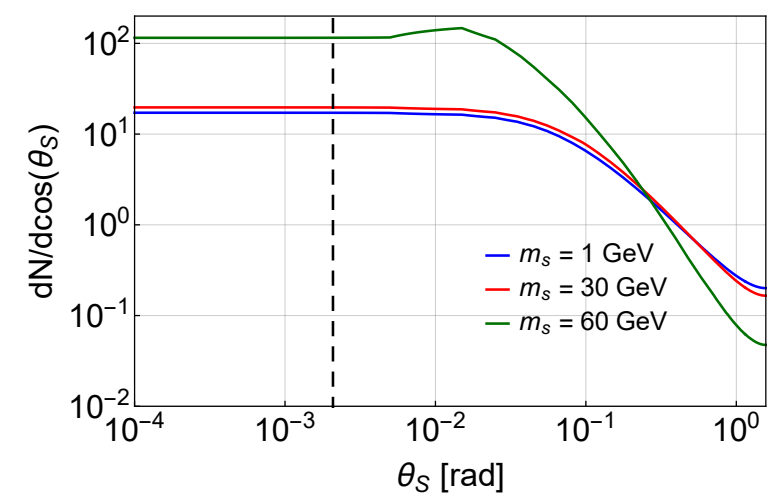

Figure 4. The angular distribution of scalars for different scalar masses. The distribution is symmetric with respect to $\pi / 2$ (right vertical axis). The vertical dashed line corresponds to $\theta_{S}=$ $\theta_{\mathrm{FASER} 2}$. The estimate is made under the assumption that Higgs bosons fly along the beam axis (see text for details).
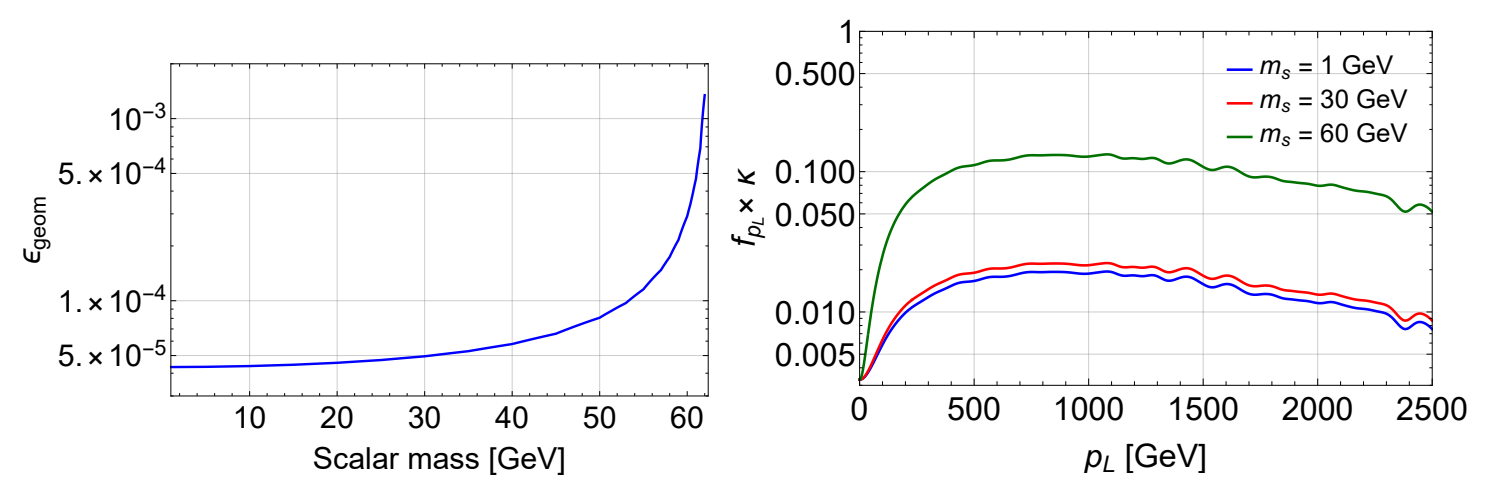

Figure 5. Left panel: Geometric acceptance of scalars at FASER 2 obtained using $p_{L}$ distribution of Higgs bosons, see eq. (2.8). Right panel: the distribution function of Higgs bosons by the longitudinal momentum $p_{L}$ multiplied by the enhancement factor $\kappa(2.10)$ for the masses of the scalar $m_{S}=0,50$ and $60 \mathrm{GeV}$.

under the assumption that $p_{L}$ and $p_{T}$ distributions of Higgs boson are independent is

$$
f_{h \rightarrow \text { FASER }} \approx \epsilon_{\text {geom }}^{\max }=\frac{1}{2} \int_{0}^{\infty} f_{p_{L}} d p_{L} \int_{0}^{p_{L}^{h} \theta_{\mathrm{FASER}}} f_{p_{T}} d p_{T} \approx 1.1 \cdot 10^{-3},
$$

where a factor $1 / 2$ comes from the fact that we do not take into account Higgs bosons that fly in the opposite direction to FASER. This number represents a maximally possible geometric acceptance.

\subsection{Decay of scalars}

The decay width and branching $S \rightarrow$ visible is determined based on the (extended) results of ref. [10] (see figure 1). For these masses, all major decay channels have $\geqslant 2$ charged tracks and therefore it is reasonable to assume that $\mathrm{BR}_{\text {visible }}=100 \%$ and that every decay is 
reconstructable with 100\% efficiency. The verification of this assumption requires detailed studies beyond the scope of this paper.

So far we have kept the decay probability at its maximum (corresponding to $l_{\text {decay }}=L$ ). This condition would give a line in the $\left(m_{S}, \theta\right)$ plane. To determine the transversal shape of the sensitivity region, we need to vary $\theta$ and take into account the $\gamma$ factor of the scalar, $\gamma_{S}$. The energy of a scalar is proportional to the energy $\left(p_{L}\right)$ of the corresponding Higgs boson:

$$
E_{S}=\frac{E_{h}}{2}\left(1+\beta_{S}^{\prime} \beta_{h} \cos \left(\theta_{S}\right)\right) \approx \frac{E_{h}}{2}\left(1+\beta_{S}^{\prime} \beta_{h}\right),
$$

where we have taken into account that the FASER detector is almost co-aligned with the beam axis and therefore $\theta_{S} \approx 0$ and neglected the $p_{T}$ distribution of the Higgs boson. The average energy of the scalar is determined by weighting the Higgs distribution $f_{p_{L}}$ with the function $\kappa$, defined in eq. (2.10). In this way, only the energies of scalars flying into the FASER 2 solid angle are considered. The resulting $\left\langle E_{S}\right\rangle$ as a function of the scalar mass is shown in figure 7 (central panel). One can see that the $\gamma$ factor ranges from $\mathcal{O}(100)$ for small masses down to $\mathcal{O}(10)$ for $m_{S} \approx m_{h} / 2$.

Let us now improve the estimate (2.5) of the maximally possible value of the decay probability $P_{\text {decay }}^{(\max )}$. The value $(2.5)$ is obtained using the average energy $\left\langle E_{S}\right\rangle$. Taking into account the continuous scalar spectra leads to a decrease of $P_{\text {decay }}^{(\max )}$. The averaging over the spectrum can be done using the function $\kappa f_{p_{L}}$ (shown in the right panel of figure 5):

$$
\left\langle P_{\text {decay }}^{(\max )}\right\rangle \approx \int \kappa\left(m_{S}, p_{L}\right) \cdot f_{p_{L}} \cdot P_{\text {decay }}\left(m_{S}, \theta^{2}, E_{S}\right) d p_{L}
$$

As is demonstrated by figure $5, \kappa \cdot f_{p_{L}}$ have similar flat shape for wide range of momenta for all possible scalar masses. We can always adjust the appropriate $\theta^{2}$ value to maximize the probability, and independently on the mass we get

$$
\left\langle P_{\text {decay }}^{(\max )}\right\rangle \simeq 3.2 \cdot 10^{-3}
$$

Substituting this value for the decay probability, as well as the number of Higgs bosons produced by the fiducial branching ratio (2.3), $\epsilon_{\text {geom }}$ (figure 5, left panel) into eq. (2.1), one can compute the improved analytic estimate for the maximal number of decay events inside the FASER 2 detector:

$$
N_{\text {events }}^{(\max )}=N_{h} \cdot \mathrm{BR}_{\text {fid }}(h \rightarrow S S) \cdot \epsilon_{\text {geom }} \cdot\left\langle P_{\text {decay }}^{(\max )}\right\rangle
$$

It is shown in figure 6 . The behavior of $N_{\text {events }}^{(\max )}$ with the scalar mass is completely determined by $\epsilon_{\text {geom }}$. Namely, the masses $m_{S} \lesssim 30 \mathrm{GeV}$ it is a constant of the order of $\mathcal{O}(1)$, while for larger masses increases due to the behavior of the geometric acceptance.

However, these estimates warrant a more detailed sensitivity study using the realistic distribution of Higgs bosons.

\section{Results}

We simulated Higgs boson production at the LHC using MadGraph5_aMCNLO [71] and following [72], see appendix A for details. Using the $p_{L}$ and $p_{T}$ distributions of the Higgs 


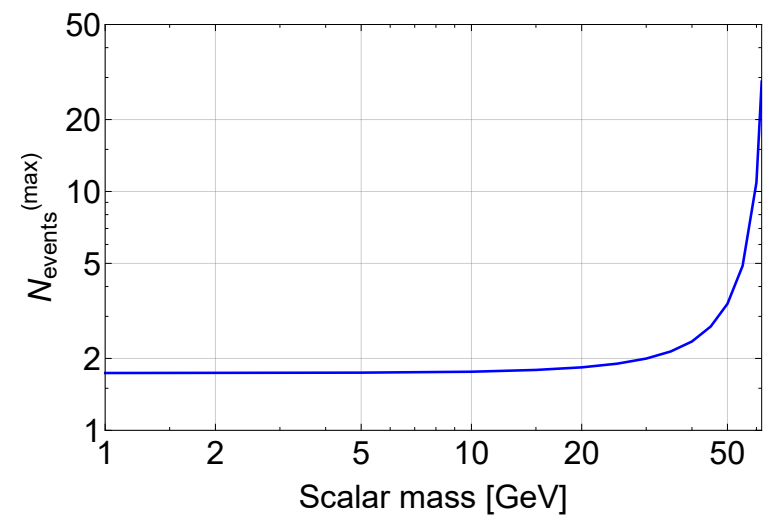

Figure 6. The analytic estimate (2.15) for the maximal number of scalar decays in FASER2 decay volume versus the scalar mass. See text for details.
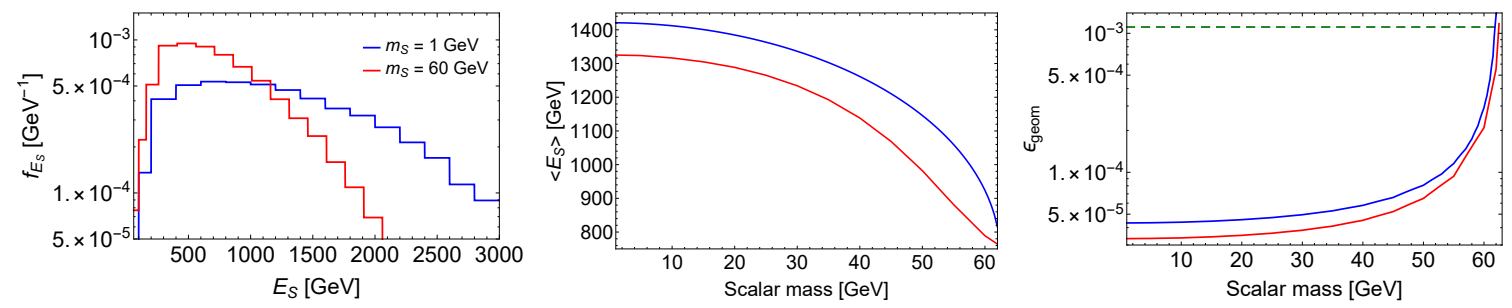

Figure 7. Properties of dark scalars flying into the FASER 2 decay volume. Left panel: energy spectrum of scalars $f_{E_{S}}=\frac{1}{N_{S}} \frac{d N_{S}}{d E_{S}}$ for different masses. Middle panel: The average energy of scalars. Right panel: The geometric acceptance $\epsilon_{\text {geom }}$ versus the scalar mass. In the middle and right panels, the blue lines denote analytic estimates obtained using the Higgs $p_{L}$ spectrum (right panel in figure 3), while the red lines show the results of more accurate estimates including the $p_{T}$ distribution of the Higgs bosons (see appendix B.2).

bosons, we derived the energy distribution of scalars $f_{E_{S}}=\frac{1}{N_{S}} \frac{d N_{S}}{d E_{S}}$ and computed the geometric acceptance $\epsilon_{\text {geom }}$, see appendix B.2.

The resulting energy distribution of scalars of particular masses traveling into the solid angle of FASER 2 is shown in figure 7 (left panel). In the same figure (middle and right panels) we compare the geometric acceptance and average energy for scalars obtained in simulations with the analytic prediction from figure 5. The simulation results lie slightly below the analytic estimate due to the $p_{T}$ distribution of Higgs bosons. The smallness of the discrepancy is related to the smallness of the ratio $\left\langle p_{T}\right\rangle /\left\langle p_{L}\right\rangle$ for the Higgs bosons. Next, we compute the number of scalars traveling through the FASER 2 fiducial volume and estimate the number of decay events, using eq. (2.1) with the decay probability $P_{\text {decay }}$ averaged over the energies of scalars flying in the direction of the experiment. The resulting sensitivity region is shown in figure 8. We assume background free experiment and therefore determine the sensitivity as a region that includes at least 2.3 events. With the current configuration of FASER 2, one can expect to see any events only in the region around $50-60 \mathrm{GeV}$. The green line follows from the analytic estimate (2.1) in which the geometric acceptance and average energy from figure 7 , whereas the blue contours are based on the more accurate 


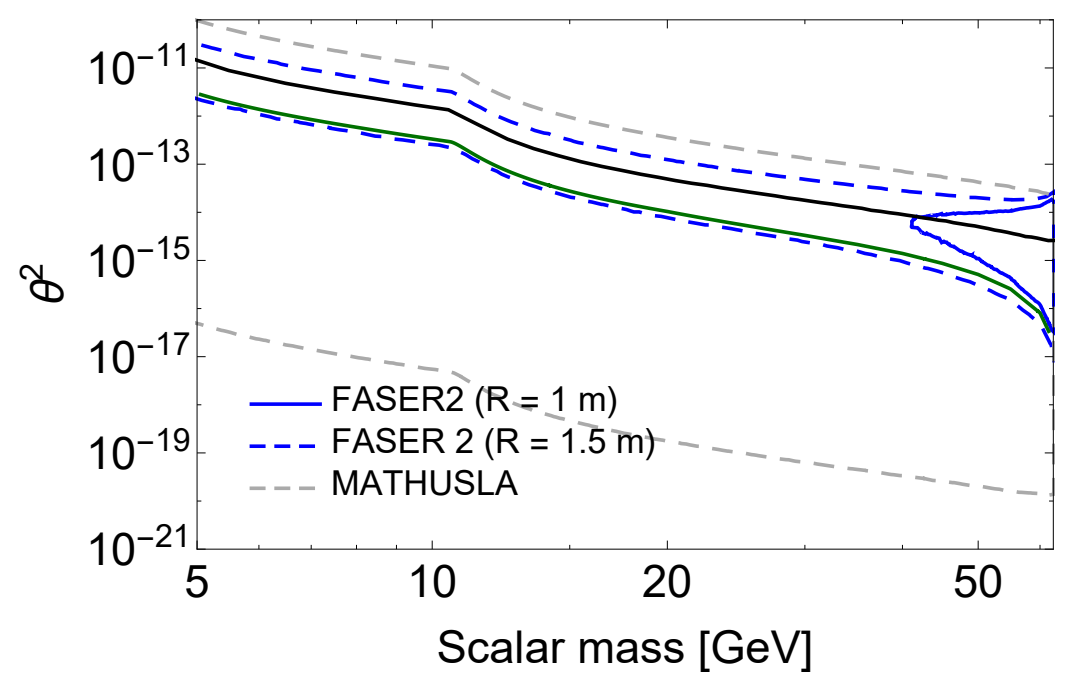

Figure 8. Sensitivity of the FASER 2 to scalars produced in decays of Higgs bosons. Blue solid line encloses the region where one expects to observe at least 2.3 events, given the current configuration of the experiment (the radius of the decay vessel $R=1 \mathrm{~m}$ ). A modest increase of the geometric acceptance (by changing the radius to $R=1.5 \mathrm{~m}$ ) allows probing an order-of-magnitude-wide stripe for all masses (between blue dashed lines). The black solid line shows parameters for which $l_{\text {decay }}=L$ (used for our analytic estimates). Gray dashed line shows upper and lower regions of the MATHUSLA200 experiment where similar production from the Higgs bosons is possible (partially based on [61]). The green line is an analytic estimate, see text for details. Sensitivity estimates assume the $100 \%$ efficiency of the reconstruction of decay products but take into account geometric acceptance. The branching ratio $\operatorname{BR}(h \rightarrow S S)$ is taken at the level of $5 \%$.

estimate using the scalar energy spectrum (see appendix B.2). A slight difference between these estimates is caused by the difference between the value of $\left.P_{\text {decay }}\left(\left\langle l_{\text {decay }}\right\rangle\right)\right)$ and $\left\langle P_{\text {decay }}\right\rangle$ where in the former case $l_{\text {decay }}$ is evaluated for $\left\langle E_{S}\right\rangle$ and in the latter case one averages $P_{\text {decay }}$ over the energy distribution.

Our results lead to an important conclusion regarding a configuration of the FASER 2. Figure 6 shows that the FASER 2 in its current configuration (as shown in table 1) will not detect any events for $m_{N} \lesssim 40 \mathrm{GeV}$ (region to the left of the blue solid line). However, a modest (factor of 2) increase in the geometrical acceptance would allow probing the whole mass range few $\mathrm{GeV} \lesssim m_{S} \lesssim m_{h} / 2$, as demonstrated by the blue dashed line in figure 8 . This increase can be achieved for example by increasing the radius of the FASER 2 from 1 meter to 1.5 meters, which is allowed by the size of the TI12 tunnel where the experiment will be located. The angular distribution of scalars is flat for relevant angles, see figure 4, which provides the desired conclusion.

\section{Conclusion}

In this work, we presented the analytic estimates for the sensitivity of the FASER 2 experiment for the most general scalar portal model including renormalizable operators only. The estimates were verified by MadGraph simulations, showing a very good agreement. 
Majority of previous works on the subject $[10-14,17,19]$ considered the models of the scalar where the term $\alpha_{1} S H^{\dagger} H$ was absent in the Lagrangian (1.1) (assuming a $\mathbb{Z}_{2}$ symmetry $S \rightarrow-S$ ). In this case, two scalar couplings $\theta$ and $\alpha$ in the effective Lagrangian (1.2) become related (and should both be small to satisfy bounds from the previous experiments).

However, if cubic and quartic couplings ( $\alpha_{1}$ and $\alpha_{2}$ in the Lagrangian (1.1)) are independent and both non-zero, the resulting triple coupling between Higgs and two scalars can be quite sizeable. Indeed, the main experimental bound on its value is the branching fraction of the invisible Higgs decay (assuming it is saturated by the $h \rightarrow S S$ process). The current bound on the invisible branching ratio $\mathrm{BR}_{\text {inv }}<0.19$ (at 95\%CL, [73]). Future runs of the LHC are expected to probe this branching at the level 0.1 or slightly below.

As a result, for the experimentally admissible values of the parameter $\alpha$, the production of scalars at the LHC from the decays of the Higgs boson $(h \rightarrow S S)$ dominates significantly over all other production channels. This makes the production and decay of a scalar controlled by independent coupling constants. This independence qualitatively changes the behavior of the sensitivity curves of the LHC-based intensity frontier experiments (MATHUSLA, FASER, CODEX-b). Indeed, normally the sensitivity of the intensity frontier experiments has a lower bound, defined by the minimal number of events in the detector, depends both on the production and decay, and an upper bound, defined by the requirement that new particles should not decay before reaching the detector (the lifetime gets smaller with mass). Their intersection often defines the maximal mass of scalar that can be probed [51]. In our case, the maximal mass is determined solely by the kinematics $\left(m_{S} \leq m_{h} / 2\right)$. However, as the geometrical acceptance drops with the decrease of the scalar's mass (see left panel of figure 5) while the number of produced scalars is massindependent, for a given geometry there can be a minimal mass that can be probed (cf. the blue solid line in figure 8).

For our analysis, we assumed that the invisible Higgs decay has a significant contribution from $h \rightarrow S S$ and, as an example, adopted a fiducial branching fraction $\operatorname{BR}(h \rightarrow S S)$ at the level of $5 \%$. We show that in this case, even if the HL-LHC does not discover invisible Higgs decay, the FASER 2 experiment is capable of discovering dark scalars with masses of $40 \mathrm{GeV} \lesssim m_{S} \lesssim m_{h} / 2$. Moreover, if its geometric acceptance is increased by a factor $\sim 2$, FASER 2 will have sensitivity for all scalar masses from $m_{h} / 2$ down to a few $\mathrm{GeV}$ and even lower, where the production from $B$ mesons starts to contribute. This can be achieved, for example, by scaling the radius of the detector from 1 meter to 1.5 meters.

Another possibility would be to put the detector closer to the interaction point, in which case the number of particles, counterintuitively, increases as $L^{3}$ ( $L^{2}$ dependence comes from the increase of the solid angle $\Omega_{\text {FASER }}$ and an extra factor comes from the $L$-dependence on the maximal decay probability, eq. (2.5)). The latter effect is due to the independence of the decay probability on the coupling $\alpha$ controlling production and is specific for the model in question. As suggested e.g. in the original FASER paper [55], another possibility would be to put the detector at 150 meters behind the TAN neutral particle absorber [74]. Such a position, however, would suffer from a high background and therefore our estimates (performed under the background-free assumption) will not be valid. Another option suggested in [55] does not increase acceptance. Indeed, it was 

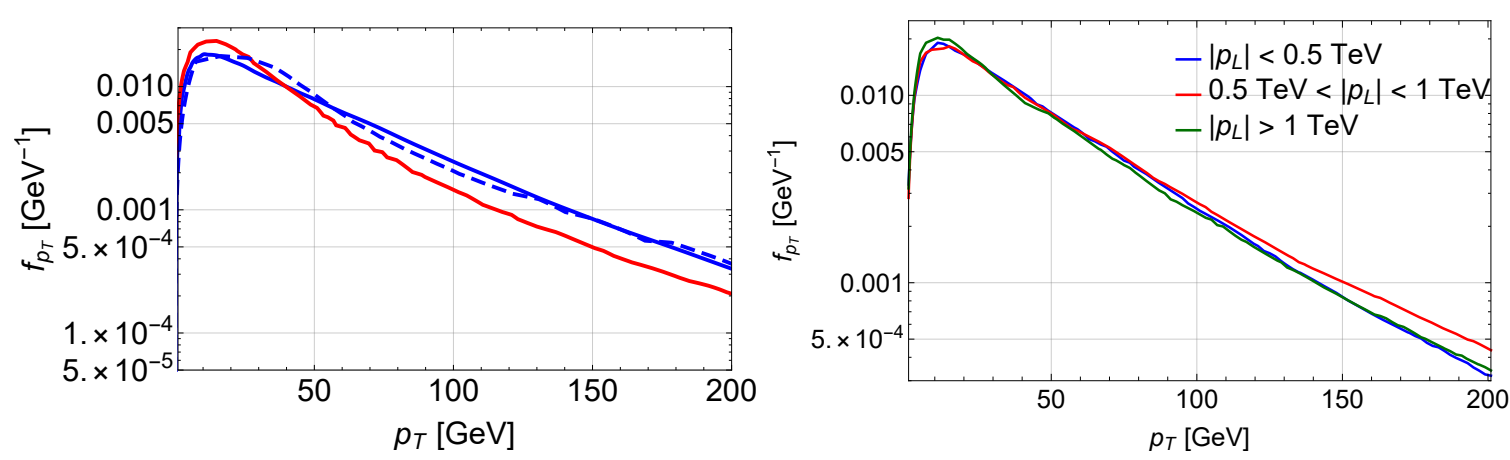

Figure 9. Left panel: a comparison of $p_{T}$ spectra of Higgs bosons obtained in our simulations (solid blue line) with the spectra from [72] (dashed blue line) and [75] (red line). See text for details. Right panel: the $p_{T}$ distribution of Higgs bosons for different domains of $\left|p_{L}\right|$.

proposed to use a hollow cylinder around the beam axis, with an inner angle around $1 \mathrm{mrad}$ (the size being dictated by the position of TAS quadrupole magnets shield) and the outer size of about $2 \mathrm{mrad}$. Such a detector would have a factor of a few lower geometric acceptance. Of course, such a detector would be too complicated and cumbersome, so its realistic version, occupying only a small sector in the azimuthal angle $\Delta \phi$, would have its geometric acceptance further reduced by $\Delta \phi / 2 \pi$.

\section{Acknowledgments}

We thank J. Boyd, O. Mattelaer, and S. Trojanowski for fruitful discussions. This project has received funding from the European Research Council (ERC) under the European Union's Horizon 2020 research and innovation program (GA 694896), from the Netherlands Science Foundation (NWO/OCW). O.R. also acknowledges support from the Carlsberg Foundation.

\section{A Higgs boson distribution}

For our estimate we used a number of Higgs bosons for HL LHC $N_{h}=1.7 \cdot 10^{8}$. To find Higgs bosons momentum distribution, we simulated Higgs boson production at the LHC using MadGraph5_aMCNLO [71] and following [72]. Using the generated events, we find that the $p_{T}$ distribution depends only weakly on $p_{L}$, see figure 9 . Therefore, the correlations between $p_{T}$ and $p_{L}$ distributions can be neglected, and the double distribution of Higgs bosons in $p_{T}, p_{L}$ can be approximated by the product of $p_{T}$ and $p_{L}$ single distributions.

We validated our simulation by comparing the $p_{T}$ spectrum of the Higgs bosons with the theoretical spectra from [72] and [75], in which the spectrum was obtained using POWHEG, see figure 9. Our results agree well with [72], while there is a discrepancy with [75] in the domain of high $p_{T}$. However, the discrepancy is not significant; in particular, the amounts of Higgs bosons flying in the direction of FASER 2 experiment calculated using our distribution and the distribution from [75] differs by no more than $30 \%$. 
For each simulated event we calculated $\kappa\left(\theta_{h}, \gamma_{h}\right)$ and the energy $E_{S}\left(\theta_{h}, \gamma_{h}\right)$ of a scalar traveling into the solid angle of FASER 2. The $\langle\kappa\rangle$ is then obtained as the arithmetic mean, while the energy distribution is obtained as the weighted distribution, where the energy $E_{S}\left(\theta_{h}, \gamma_{h}\right)$ has the corresponding weight $\kappa\left(\theta_{h}, \gamma_{h}\right)$.

\section{B Distributions}

\section{B.1 Kinematics in laboratory frame}

Consider the relation between the laboratory frame angle $\theta_{S}$ and the rest frame angle $\theta_{S}^{\prime}$ :

$$
\tan \left(\theta_{S}\right)=\frac{1}{\gamma_{h}} \frac{\beta_{S}^{\prime} \sin \left(\theta_{S}^{\prime}\right)}{\beta_{S}^{\prime} \cos \left(\theta_{S}^{\prime}\right)+\beta_{h}}
$$

Let us introduce two functions

$$
f_{ \pm}\left(\theta_{S}\right)=-\frac{\beta_{h} \gamma_{h}^{2} \tan ^{2}\left(\theta_{S}\right) \pm \sqrt{\beta_{S}^{\prime 2}+\left(\beta_{S}^{\prime 2}-\beta_{h}^{2}\right) \gamma_{h}^{2} \tan ^{2}\left(\theta_{S}\right)}}{\beta_{S}^{\prime}\left(1+\gamma_{h}^{2} \tan ^{2}\left(\theta_{S}\right)\right)},
$$

representing the solution of eq. (B.1) in terms of $\cos \left(\theta_{S}^{\prime}\right)$ for given parameters $\beta_{h}, \beta_{S}$. In order to express $\cos \left(\theta_{S}^{\prime}\right)$ from eq. (B.1), we find first the values of $\theta_{S}$ where the functions (B.2) become complex. These are $\theta_{S, \max }<\theta_{S}<\pi-\theta_{S, \max }$, defined as

$$
\theta_{S, \max }=\arctan \left[\frac{\beta_{S}^{\prime}}{\gamma_{h} \sqrt{\beta_{h}^{2}-\beta_{S}^{\prime 2}}}\right]
$$

They are always real as long as $\beta_{h} / \beta_{S}^{\prime}<1$. Next, we can construct the physical solution $\cos \left(\theta_{S}^{\prime}\right)$ requiring the solutions (B.2) to cover all the domain of the definition of the cosine, $\cos \left(\theta_{S}^{\prime}\right) \in[-1,1]$. For $\beta_{h} / \beta_{S}^{\prime}<1$ it is

$$
\begin{aligned}
\cos \left(\theta_{S}^{\prime}\right) & =\left\{\begin{array}{l}
f_{-}\left(\theta_{S}\right), 0<\theta_{S}<\pi / 2, \\
f_{+}\left(\theta_{S}\right), \quad \pi / 2<\theta_{S}<\pi
\end{array}\right. \\
& =-\frac{\beta_{h} \gamma_{h}^{2} \sin ^{2}\left(\theta_{S}\right)-\cos \left(\theta_{S}\right) \sqrt{\beta_{S}^{\prime 2} \cos ^{2}\left(\theta_{S}\right)+\left(\beta_{S}^{\prime 2}-\beta_{h}^{2}\right) \gamma_{h}^{2} \sin ^{2}\left(\theta_{S}\right)}}{\beta_{S}^{\prime} \cos ^{2}\left(\theta_{S}\right)+\gamma_{h}^{2} \sin ^{2}\left(\theta_{S}\right)}
\end{aligned}
$$

For $\beta_{h}>\beta_{S}^{\prime}$ both the solutions $f_{ \pm}$exist in the domain $\theta_{S}<\theta_{S \text {, max }}$.

Let us now find the function $\kappa$. By the definition, $\kappa=\left|d \cos \left(\theta_{S}^{\prime}\right) / d \cos \left(\theta_{S}\right)\right|$. In the case $\beta_{h}<\beta_{S}^{\prime}$ it is simply given by the derivative of (B.4), while for the case $\beta_{h}>\beta_{S}^{\prime}$ it reads

where

$$
\kappa=\left|\frac{d f_{+}\left(\theta_{S}\right)}{d \cos \left(\theta_{S}\right)}\right|+\left|\frac{d f_{-}\left(\theta_{S}\right)}{d \cos \left(\theta_{S}\right)}\right|=\frac{d g\left(\theta_{S}\right)}{d \cos \left(\theta_{S}\right)}
$$

$$
g\left(\theta_{S}\right)=\left|2 \cos \left(\theta_{S}\right) \frac{\sqrt{\beta_{S}^{\prime 2} \cos ^{2}\left(\theta_{S}\right)+\left(\beta_{S}^{\prime 2}-\beta_{h}^{2}\right) \gamma_{h}^{2} \sin ^{2}\left(\theta_{S}\right)}}{\beta_{S}^{\prime}\left(\cos ^{2}\left(\theta_{S}\right)+\gamma_{h}^{2} \sin ^{2}\left(\theta_{S}\right)\right)}\right|
$$

In particular, in the domain $\theta_{S} \ll \theta_{S, \text { max }}$ for $\beta_{h}>\beta_{S}^{\prime}$ we have

$$
g\left(\theta_{S}\right) \approx 2-\frac{\theta_{S}^{2}\left(\beta_{h}^{2}+\beta_{S}^{\prime 2}\right)}{\beta_{S}^{\prime 2}} \Rightarrow \kappa \approx \frac{2 \gamma_{h}^{2}\left(\beta_{S}^{\prime 2}+\beta_{h}^{2}\right)}{\beta_{S}^{\prime 2}}
$$




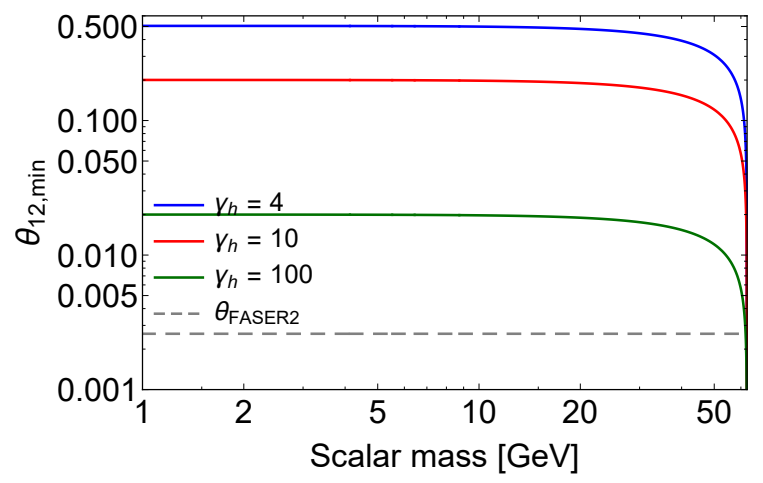

Figure 10. The minimal angle (B.9) between two scalars produced in the decay $H \rightarrow S S$ versus the scalar mass $m_{S}$ for particular values of the $\gamma$ factor of the Higgs boson.

\section{B.2 Distribution of scalars over energies and polar angles}

The double differential distribution $f_{E_{S}, \theta_{S}}$ of scalars produced in the decay $h \rightarrow S S$ has been calculated in the following way. Consider a differential branching ratio for a Higgs bosons flying in the direction $\theta_{h}, \phi_{h}$ :

$$
d \operatorname{Br}(h \rightarrow S S)=\frac{1}{2} \frac{1}{(2 \pi)^{2}} \frac{|\mathcal{M}|^{2}}{2 \Gamma_{h, \text { rest }} m_{h}} \frac{d^{3} \boldsymbol{p}_{S_{1}}}{2 E_{S_{1}}} \int \frac{d^{3} \boldsymbol{p}_{S_{2}}}{2 E_{S_{2}}} \delta^{4}\left(p_{h}-p_{S_{1}}-p_{S_{2}}\right),
$$

where $\mathcal{M}$ is the invariant matrix element of the process $h \rightarrow S S$ (independent on momenta for $1 \rightarrow 2$ process), $\boldsymbol{p}_{S_{1,2}}$ are momenta of two produced scalars.

Two scalars are indistinguishable (extra factor $1 / 2$ in eq. (B.8)) and after phase space integration we would lose the information about the relative distribution of the two scalars. In particular we cannot trace whether one or both scalars simultaneously could enter the FASER 2 decay volume which could lead to underestimate of the number of events by as much as a factor of 2 . However, because of the small angular size of the FASER 2 experiment, the fraction of events with two $S$ s flying into the detector's fiducial volume is negligibly small. Indeed, the minimal angle $\theta_{12 \text {,min }}$ between two scalars produced in the decay $h \rightarrow S S$ is given by

$$
\sin \left(\theta_{12, \min }\right)=\frac{2 m_{h}^{2} \beta_{h} \sqrt{\gamma_{h}^{2}-1}}{m_{h}^{2} \gamma_{h}^{2}-4 m_{S}^{2}}
$$

It is larger than $\theta_{\text {FASER }} \approx 2.6 \cdot 10^{-3}$ for all values of $\gamma_{h}$ reachable at the LHC for $m_{S} \lesssim$ $62 \mathrm{GeV}$, see figure 10. After the integration over $\mathbf{p}_{S_{2}}$, replacing $S_{1} \rightarrow S$ we get

$$
d \operatorname{Br}(h \rightarrow S S)=\frac{d^{3} \mathbf{p}_{S}}{8(2 \pi)^{2}} \frac{|\mathcal{M}|^{2}}{4 \Gamma_{h, \text { rest }} m_{h} E_{S}} \delta\left(m_{h}^{2}-2 E_{S} E_{h}+2\left|\boldsymbol{p}_{S}\right|\left|\boldsymbol{p}_{h}\right| \cos (\alpha)\right),
$$

where

$$
\cos (\alpha)=\cos \left(\phi_{h}\right) \sin \left(\theta_{h}\right) \sin \left(\theta_{S}\right)+\cos \left(\theta_{h}\right) \cos \left(\theta_{S}\right)
$$

is the angle between the Higgs boson and the scalar. Rewriting the scalar phase space volume as $d^{3} \boldsymbol{p}_{S}=\sin \left(\theta_{S}\right) d \theta_{S} E_{S} \sqrt{E_{S}^{2}-m_{S}^{2}} d E_{S} d \phi_{S}$, for the distribution in the energy and 
polar angle is given by

$$
\begin{aligned}
f_{\theta_{S}, E_{S}}= & \frac{1}{\mathrm{BR}_{h \rightarrow S S}} \frac{d \mathrm{BR}(h \rightarrow S S)}{d \theta_{S} d E_{S}} \\
= & 2 \pi \frac{\sin \left(\theta_{S}\right) E_{S} \sqrt{E_{S}^{2}-m_{S}^{2}}}{\operatorname{Br}(h \rightarrow S S)} \int \frac{d \phi_{h}}{2 \pi} d E_{h} d \theta_{h} f_{\theta_{h}, E_{h}} \frac{d^{3} \operatorname{Br}(h \rightarrow S S)}{d^{3} \boldsymbol{p}_{S}} \\
= & \frac{m_{h} \sqrt{E_{S}^{2}-m_{S}^{2}}}{\left|\boldsymbol{p}_{S, \text { rest }}\right|} \sin \left(\theta_{S}\right) I\left[\theta_{S}, E_{S}\right],
\end{aligned}
$$

where $f_{\theta_{h}, E_{h}}$ is the double differential distribution of the Higgs bosons obtained in simulations, and

$$
I\left[\theta_{S}, E_{S}\right]=\frac{1}{2 \pi} \int d \phi_{h} d \theta_{h} d E_{h} f_{\theta_{h}, E_{h}} \delta\left(m_{h}^{2}-2 E_{S} E_{h}+2\left|\boldsymbol{p}_{S}\right|\left|\boldsymbol{p}_{h}\right| \cos (\alpha)\right)
$$

Having the distribution function (B.12), the number of events may be determined as

$$
N_{\text {det }}=N_{S} \cdot \operatorname{BR}(h \rightarrow S S) \cdot \int d \theta_{S} d E_{S} f_{\theta_{S}, E_{S}} P_{\text {decay }}\left(E_{S}\right)
$$

Open Access. This article is distributed under the terms of the Creative Commons Attribution License (CC-BY 4.0), which permits any use, distribution and reproduction in any medium, provided the original author(s) and source are credited.

\section{References}

[1] I. Brivio and M. Trott, The Standard Model as an Effective Field Theory, Phys. Rept. 793 (2019) 1 [arXiv: 1706. 08945] [INSPIRE].

[2] J. McDonald, Gauge singlet scalars as cold dark matter, Phys. Rev. D 50 (1994) 3637 [hep-ph/0702143] [INSPIRE].

[3] C.P. Burgess, M. Pospelov and T. ter Veldhuis, The minimal model of nonbaryonic dark matter: A singlet scalar, Nucl. Phys. B 619 (2001) 709 [hep-ph/0011335] [INSPIRE].

[4] B. Patt and F. Wilczek, Higgs-field portal into hidden sectors, hep-ph/0605188 [INSPIRE].

[5] D. O'Connell, M.J. Ramsey-Musolf and M.B. Wise, Minimal Extension of the Standard Model Scalar Sector, Phys. Rev. D 75 (2007) 037701 [hep-ph/0611014] [INSPIRE].

[6] A. Djouadi, A. Falkowski, Y. Mambrini and J. Quevillon, Direct Detection of Higgs-Portal Dark Matter at the LHC, Eur. Phys. J. C 73 (2013) 2455 [arXiv:1205.3169] [INSPIRE].

[7] D. Curtin et al., Exotic decays of the 125 GeV Higgs boson, Phys. Rev. D 90 (2014) 075004 [arXiv: 1312.4992] [INSPIRE].

[8] S. Alekhin et al., A facility to Search for Hidden Particles at the CERN SPS: the SHiP physics case, Rept. Prog. Phys. 79 (2016) 124201 [arXiv:1504.04855] [INSPIRE].

[9] G. Arcadi, A. Djouadi and M. Raidal, Dark Matter through the Higgs portal, Phys. Rept. 842 (2020) 1 [arXiv: 1903.03616] [INSPIRE].

[10] I. Boiarska, K. Bondarenko, A. Boyarsky, V. Gorkavenko, M. Ovchynnikov and A. Sokolenko, Phenomenology of GeV-scale scalar portal, JHEP 11 (2019) 162 [arXiv:1904.10447] [INSPIRE]. 
[11] C. Bird, P. Jackson, R.V. Kowalewski and M. Pospelov, Search for dark matter in $b \rightarrow s$ transitions with missing energy, Phys. Rev. Lett. 93 (2004) 201803 [hep-ph/0401195] [INSPIRE].

[12] B. Batell, M. Pospelov and A. Ritz, Multi-lepton Signatures of a Hidden Sector in Rare B Decays, Phys. Rev. D 83 (2011) 054005 [arXiv:0911.4938] [INSPIRE].

[13] F. Bezrukov and D. Gorbunov, Light inflaton Hunter's Guide, JHEP 05 (2010) 010 [arXiv: 0912.0390] [INSPIRE].

[14] J.D. Clarke, R. Foot and R.R. Volkas, Phenomenology of a very light scalar (100 MeV $\leq m_{h} \leq 10 \mathrm{GeV}$ ) mixing with the SM Higgs, JHEP 02 (2014) 123 [arXiv:1310.8042] [INSPIRE].

[15] K. Schmidt-Hoberg, F. Staub and M.W. Winkler, Constraints on light mediators: confronting dark matter searches with B physics, Phys. Lett. B 727 (2013) 506 [arXiv: 1310.6752] [INSPIRE].

[16] J.A. Evans, Detecting Hidden Particles with MATHUSLA, Phys. Rev. D 97 (2018) 055046 [arXiv: 1708.08503] [INSPIRE].

[17] F. Bezrukov, D. Gorbunov and I. Timiryasov, Uncertainties of hadronic scalar decay calculations, arXiv: 1812.08088 [INSPIRE].

[18] A. Monin, A. Boyarsky and O. Ruchayskiy, Hadronic decays of a light Higgs-like scalar, Phys. Rev. D 99 (2019) 015019 [arXiv: 1806.07759] [InSPIRE].

[19] M.W. Winkler, Decay and detection of a light scalar boson mixing with the Higgs boson, Phys. Rev. D 99 (2019) 015018 [arXiv:1809.01876] [INSPIRE].

[20] C. Frugiuele, E. Fuchs, G. Perez and M. Schlaffer, Relaxion and light (pseudo)scalars at the HL-LHC and lepton colliders, JHEP 10 (2018) 151 [arXiv:1807.10842] [INSPIRE].

[21] A.J. Helmboldt and M. Lindner, Prospects for three-body Higgs boson decays into extra light scalars, Phys. Rev. D 95 (2017) 055008 [arXiv:1609.08127] [INSPIRE].

[22] M.B. Voloshin, Once Again About the Role of Gluonic Mechanism in Interaction of Light Higgs Boson with Hadrons, Sov. J. Nucl. Phys. 44 (1986) 478 [InSPIRE].

[23] S. Raby and G.B. West, The Branching Ratio for a Light Higgs to Decay Into $\mu^{+} \mu^{-}$Pairs, Phys. Rev. D 38 (1988) 3488 [inSPIRE].

[24] T.N. Truong and R.S. Willey, Branching Ratios for Decays of Light Higgs Bosons, Phys. Rev. D 40 (1989) 3635 [INSPIRE].

[25] J.F. Donoghue, J. Gasser and H. Leutwyler, The Decay of a Light Higgs Boson, Nucl. Phys. B 343 (1990) 341 [INSPIRE].

[26] R.S. Willey and H.L. Yu, The Decays $K^{ \pm} \rightarrow \pi^{ \pm} \ell^{+} \ell^{-}$and Limits on the Mass of the Neutral Higgs Boson, Phys. Rev. D 26 (1982) 3287 [InSPIRE].

[27] R.S. Willey, Limits on Light Higgs Bosons From the Decays $K^{ \pm} \rightarrow \pi^{ \pm} \ell^{-} \ell^{+}$, Phys. Lett. B 173 (1986) 480 [INSPIRE].

[28] B. Grzadkowski and P. Krawczyk, Higgs particle effects in flavor changing transitions, Z. Phys. C 18 (1983) 43 [INSPIRE].

[29] H. Leutwyler and M.A. Shifman, Light Higgs Particle in Decays of K and $\eta$ Mesons, Nucl. Phys. B 343 (1990) 369 [INSPIRE]. 
[30] H.E. Haber, A.S. Schwarz and A.E. Snyder, Hunting the Higgs in B Decays, Nucl. Phys. B 294 (1987) 301 [INSPIRE].

[31] R.S. Chivukula and A.V. Manohar, Limits on a light Higgs boson, Phys. Lett. B 207 (1988) 86 [Erratum ibid. B 217 (1989) 568] [INSPIRE].

[32] A. Fradette, M. Pospelov, J. Pradler and A. Ritz, Cosmological beam dump: constraints on dark scalars mixed with the Higgs boson, Phys. Rev. D 99 (2019) 075004 [arXiv: 1812.07585] [INSPIRE].

[33] A. Fradette and M. Pospelov, BBN for the LHC: constraints on lifetimes of the Higgs portal scalars, Phys. Rev. D 96 (2017) 075033 [arXiv:1706.01920] [InSPIRE].

[34] CMS collaboration, Search for invisible decays of a Higgs boson produced through vector boson fusion in proton-proton collisions at $\sqrt{s}=13 \mathrm{TeV}$, Phys. Lett. B 793 (2019) 520 [arXiv: 1809.05937] [INSPIRE].

[35] ATLAS collaboration, Search for invisible Higgs boson decays in vector boson fusion at $\sqrt{s}=13 \mathrm{TeV}$ with the ATLAS detector, Phys. Lett. B 793 (2019) 499 [arXiv:1809.06682] [INSPIRE].

[36] CHARM collaboration, Search for Axion Like Particle Production in 400-GeV Proton-Copper Interactions, Phys. Lett. 157B (1985) 458 [INSPIRE].

[37] KTEV collaboration, Search for the Decay $K_{L} \rightarrow \pi^{0} \mu^{+} \mu^{-}$, Phys. Rev. Lett. 84 (2000) 5279 [hep-ex/0001006] [INSPIRE].

[38] E949 collaboration, New measurement of the $K^{+} \rightarrow \pi^{+} \nu \bar{\nu}$ branching ratio, Phys. Rev. Lett. 101 (2008) 191802 [arXiv:0808.2459] [INSPIRE].

[39] BNL-E949 collaboration, Study of the decay $K^{+} \rightarrow \pi^{+} \nu \bar{\nu}$ in the momentum region $140<P_{\pi}<199 \mathrm{MeV} / c$, Phys. Rev. D 79 (2009) 092004 [arXiv:0903.0030] [InSPIRE].

[40] Belle collaboration, Search for the dark photon and the dark Higgs boson at Belle, Phys. Rev. Lett. 114 (2015) 211801 [arXiv:1502.00084] [INSPIRE].

[41] BeLle collaboration, Search for a light CP-odd Higgs boson and low-mass dark matter at the Belle experiment, Phys. Rev. Lett. 122 (2019) 011801 [arXiv: 1809.05222] [INSPIRE].

[42] BABAR collaboration, Search for $B \rightarrow K^{(*)} \nu \bar{\nu}$ and invisible quarkonium decays, Phys. Rev. D 87 (2013) 112005 [arXiv: 1303.7465] [INSPIRE].

[43] LHCB collaboration, Search for hidden-sector bosons in $B^{0} \rightarrow K^{* 0} \mu^{+} \mu^{-}$decays, Phys. Rev. Lett. 115 (2015) 161802 [arXiv: 1508.04094] [INSPIRE].

[44] LHCB collaboration, Search for long-lived scalar particles in $B^{+} \rightarrow K^{+} \chi\left(\mu^{+} \mu^{-}\right)$decays, Phys. Rev. D 95 (2017) 071101 [arXiv:1612.07818] [INSPIRE].

[45] CMS collaboration, Search for an exotic decay of the Higgs boson to a pair of light pseudoscalars in the final state with two muons and two b quarks in pp collisions at $13 \mathrm{TeV}$, Phys. Lett. B 795 (2019) 398 [arXiv:1812.06359] [InSPIRE].

[46] CMS collaboration, A search for pair production of new light bosons decaying into muons in proton-proton collisions at $13 \mathrm{TeV}$, Phys. Lett. B 796 (2019) 131 [arXiv:1812.00380] [INSPIRE].

[47] ATLAS collaboration, Search for invisible decays of a Higgs boson using vector-boson fusion in pp collisions at $\sqrt{s}=8 \mathrm{TeV}$ with the ATLAS detector, JHEP 01 (2016) 172 [arXiv: 1508.07869] [INSPIRE]. 


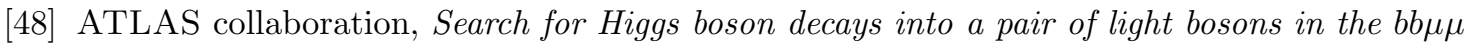
final state in pp collision at $\sqrt{s}=13 \mathrm{TeV}$ with the ATLAS detector, Phys. Lett. B 790 (2019) 1 [arXiv: 1807.00539] [INSPIRE].

[49] ATLAS collaboration, Search for the Higgs boson produced in association with a vector boson and decaying into two spin-zero particles in the $H \rightarrow a a \rightarrow 4 b$ channel in pp collisions at $\sqrt{s}=13 \mathrm{TeV}$ with the ATLAS detector, JHEP 10 (2018) 031 [arXiv:1806.07355] [INSPIRE].

[50] SHIP collaboration, A facility to Search for Hidden Particles (SHiP) at the CERN SPS, arXiv: 1504.04956 [INSPIRE].

[51] K. Bondarenko, A. Boyarsky, M. Ovchynnikov and O. Ruchayskiy, Sensitivity of the intensity frontier experiments for neutrino and scalar portals: analytic estimates, JHEP 08 (2019) 061 [arXiv: 1902.06240] [INSPIRE].

[52] V.V. Gligorov, S. Knapen, M. Papucci and D.J. Robinson, Searching for Long-lived Particles: A Compact Detector for Exotics at LHCb, Phys. Rev. D 97 (2018) 015023 [arXiv: 1708.09395] [INSPIRE].

[53] J.P. Chou, D. Curtin and H.J. Lubatti, New Detectors to Explore the Lifetime Frontier, Phys. Lett. B 767 (2017) 29 [arXiv: 1606. 06298] [INSPIRE].

[54] D. Curtin et al., Long-Lived Particles at the Energy Frontier: The MATHUSLA Physics Case, Rept. Prog. Phys. 82 (2019) 116201 [arXiv:1806.07396] [InSPIRE].

[55] J.L. Feng, I. Galon, F. Kling and S. Trojanowski, ForwArd Search ExpeRiment at the LHC, Phys. Rev. D 97 (2018) 035001 [arXiv:1708.09389] [INSPIRE].

[56] J.L. Feng, I. Galon, F. Kling and S. Trojanowski, Dark Higgs bosons at the ForwArd Search ExpeRiment, Phys. Rev. D 97 (2018) 055034 [arXiv:1710.09387] [INSPIRE].

[57] A. Berlin, S. Gori, P. Schuster and N. Toro, Dark Sectors at the Fermilab SeaQuest Experiment, Phys. Rev. D 98 (2018) 035011 [arXiv:1804.00661] [InSPIRE].

[58] SHIP collaboration, Prospects of the SHiP and NA62 experiments at CERN for hidden sector searches, PoS (NuFact2017) 139 [arXiv: 1712.01768] [INSPIRE].

[59] NA62 collaboration, Search for heavy neutral lepton production in $K^{+}$decays, Phys. Lett. B 778 (2018) 137 [arXiv: 1712.00297] [INSPIRE].

[60] M. Drewes, J. Hajer, J. Klaric and G. Lanfranchi, NA62 sensitivity to heavy neutral leptons in the low scale seesaw model, JHEP 07 (2018) 105 [arXiv: 1801.04207] [INSPIRE].

[61] J. Beacham et al., Physics Beyond Colliders at CERN: Beyond the Standard Model Working Group Report, J. Phys. G 47 (2020) 010501 [arXiv:1901.09966] [InSPIRE].

[62] P. Bechtle, S. Heinemeyer, O. Stål, T. Stefaniak and G. Weiglein, Probing the Standard Model with Higgs signal rates from the Tevatron, the LHC and a future ILC, JHEP 11 (2014) 039 [arXiv:1403.1582] [INSPIRE].

[63] J. de Blas et al., Higgs Boson Studies at Future Particle Colliders, JHEP 01 (2020) 139 [arXiv: 1905.03764] [INSPIRE].

[64] CMS collaboration, Search for an exotic decay of the Higgs boson to a pair of light pseudoscalars in the final state with two b quarks and two $\tau$ leptons in proton-proton collisions at $\sqrt{s}=13 \mathrm{TeV}$, Phys. Lett. B 785 (2018) 462 [arXiv:1805.10191] [INSPIRE].

[65] CMS collaboration, Search for an exotic decay of the Higgs boson to a pair of light pseudoscalars in the final state of two muons and two $\tau$ leptons in proton-proton collisions at $\sqrt{s}=13 \mathrm{TeV}, J H E P 11$ (2018) 018 [arXiv:1805.04865] [INSPIRE]. 
[66] FASER collaboration, FASER: ForwArd Search ExpeRiment at the LHC, arXiv: 1901.04468 [INSPIRE].

[67] FASER collaboration, Technical Proposal for FASER: ForwArd Search ExpeRiment at the $L H C$, arXiv:1812.09139 [INSPIRE].

[68] FASER collaboration, FASER's physics reach for long-lived particles, Phys. Rev. D 99 (2019) 095011 [arXiv:1811.12522] [INSPIRE].

[69] FASER collaboration, Letter of Intent for FASER: ForwArd Search ExpeRiment at the LHC, arXiv: 1811.10243 [INSPIRE].

[70] M. Cepeda et al., Report from Working Group 2, CERN Yellow Rep. Monogr. 7 (2019) 221 [arXiv: 1902.00134] [INSPIRE].

[71] J. Alwall et al., The automated computation of tree-level and next-to-leading order differential cross sections and their matching to parton shower simulations, JHEP 07 (2014) 079 [arXiv: 1405.0301] [INSPIRE].

[72] V. Hirschi and O. Mattelaer, Automated event generation for loop-induced processes, JHEP 10 (2015) 146 [arXiv:1507.00020] [INSPIRE].

[73] CMS collaboration, Search for heavy neutral leptons in events with three charged leptons in proton-proton collisions at $\sqrt{s}=13 \mathrm{TeV}$, Phys. Rev. Lett. 120 (2018) 221801 [arXiv: 1802.02965] [INSPIRE].

[74] LHCF collaboration, Measurements of longitudinal and transverse momentum distributions for neutral pions in the forward-rapidity region with the LHCf detector, Phys. Rev. D 94 (2016) 032007 [arXiv: 1507.08764] [INSPIRE].

[75] E. Bagnaschi, R.V. Harlander, H. Mantler, A. Vicini and M. Wiesemann, Resummation ambiguities in the Higgs transverse-momentum spectrum in the Standard Model and beyond, JHEP 01 (2016) 090 [arXiv: 1510.08850] [INSPIRE]. 